\title{
Role of Heterogeneity in Cancer Cells Examined through Quantitative Analysis of Single HER2 Protein Distribution and Activation Status
}

\author{
Diana Peckys ${ }^{1}$, Ulrike Korf ${ }^{2}$, Stefan Wiemann $^{2}$ and Niels de Jonge W $^{3,4}$ \\ 1. Department of Biophysics, Saarland University, D-66421 Homburg/Saar, Germany. \\ 2. Division of Molecular Genome Analysis, German Cancer Research Center, 69120 Heidelberg, \\ Germany. \\ 3. INM - Leibniz Institute for New Materials, 66123 Saarbrücken, Germany. \\ 4. Department of Physics, University of Saarland, 66123 Saarbrücken, Germany.
}

Cancer heterogeneity, a long recognized hallmark of cancer, is caused by genetic factors, such as genomic instability, and by massive epigenetic abnormalities. Because it allows cancers to progress and escape therapies, increased heterogeneity represents a substantial danger to the patient [1]. Despite this vital importance, many unknown factors persist about this complex issue and how it can be successfully combatted. The reason is that the many flavours of heterogeneity are dynamic, difficult to define, and challenging to detect and to measure. HER2 positive breast cancer, for instance, is an aggressive form of breast cancer, affecting about $20 \%$ of the patients. Since several years, it can be directly treated with targeted antibody drugs. But, even if the majority of cancer cells in a biopsy from a breast cancer patient is recognized as HER2 positive and targeted therapy is started, rare cancer stem cells and so-called "dormant" cancer cells, hiding in the primary tumour cell population, in bone marrow or at distant metastatic sides, can survive therapy, thereby conserving a life-threatening danger due to their potential to re-generate a new tumour as soon as the conditions are favourable [2].

We applied correlative light and in-situ electron microscopy to quickly detect such cells and to quantitatively examine their HER2-targetability by anti-cancer drugs. HER2 membrane receptors in breast cancer cells were labelled in a one-to-one stoichiometry with fluorescent nanoparticles, so-called quantum dots (QDs). The electron-dense cores of the QDs were visualized while bound to HER2 in intact cells in liquid using wet state environmental scanning electron microscopy (ESEM) and scanning transmission electron microscopy (STEM) detection [3]. Single molecule HER2-localization data was recorded with $3 \mathrm{~nm}$ spatial resolution from dozens of cells and hundred thousands of labelled HER2 proteins. The localized HER2 activation status was obtained via automated particle detection in the images, followed by quantitative pair correlation-, and cluster size analysis. The sample preparation was minimal and similar to procedures in fluorescence microscopy thus enabling rapid analysis $(\sim 20$ cells analysed per day). The sample preparation avoided pitfalls of possible artefacts created by sample dehydration, embedding and sectioning. Data was recorded well within the limit of radiation damage [4].

Our results confirmed a substantial heterogeneity of phenotypes within a HER2 overexpressing cancer cell population, and of mean HER2 expression levels between the individual cells. The data revealed subcellular sites without HER2 in activated, dimerized state, contrasting the average situation of these HER2 overexpressing cells dominated by HER2 homodimers. Most importantly, we found that the drug response of phenotypically distinct subpopulations, belonging to the "dormant" and to the cancer stem cell groups, differed considerably with respect to the cellular average. These distinct responses were already detectable after 3 min of drug incubation. 
In conclusion, our data show a crucial consequence of cancer cell heterogeneity, whereby it appears that an antibody-based anti-cancer drug induces different effects on minor subpopulations of cancer cells than in the average bulk cancer cell. This observation stresses the importance of studying the interaction of receptors at the molecular and at the single-cell level, while accounting for small sub-groups of cells that would normally escape any detection in method using population averages [5].

\section{References:}

[1] A Marusyk, V Almendro and K. Polyak, Nat Rev Cancer 12 (2012), p. 323.

[2] J Visvader, Nature 469 (2011), p. 312.

[3] D Peckys, U Korf and N de Jonge, Sci Adv 1 (2015), e1500165.

[4] J Hermannsdoerfer et al, Microsc Microanal 20 (2016), pp. 656-665.

[5] Research supported by the Leibniz Competition 2014. The authors thank E. Arzt for his support through INM.
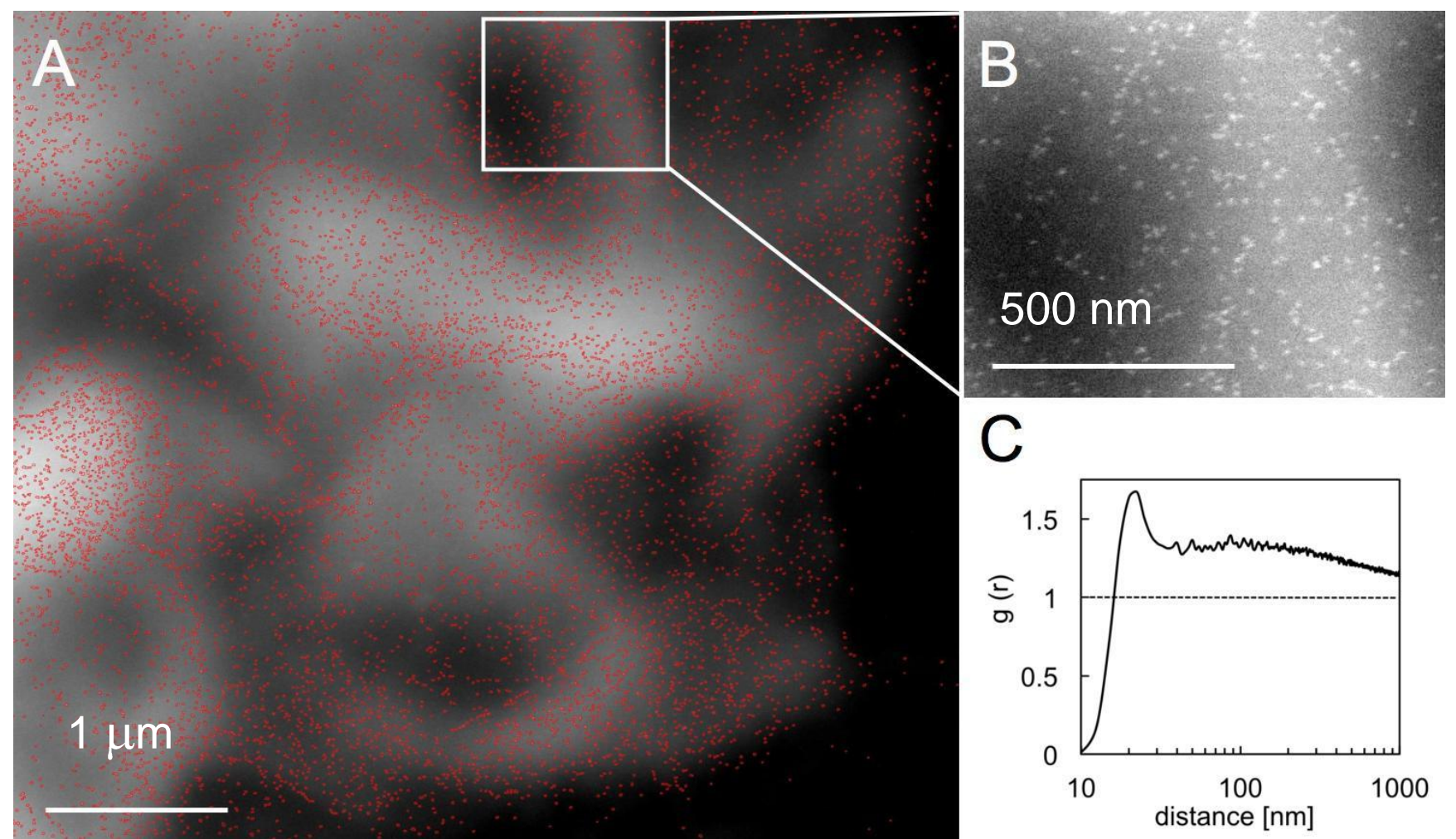

Figure 1. Quantitative analysis of HER2 positions. (A) Exemplary STEM-ESEM image $(50.000 \times$ magnification) at a ruffled region of the plasma membrane, where thousands of QD-labeled HER2 (highlighted in red) accumulate and form HER2 homodimers. (B) Selected region showing HER2 monomers, dimers, and clusters. (C) Pair correlation function $\mathrm{g}(\mathrm{r})$ calculated from all intra-label distances in A. The peak at $20 \mathrm{~nm}$ is the signature of HER2 homodimer. 